\title{
Correlation-based Initialization Algorithm for Tensor-based HSI Compression Methods
}

\author{
Rui Li, Zhibin Pan and Yang Wang
}

\begin{abstract}
Tensor decomposition (TD) is widely used in hyperspectral image (HSI) compression. The initialization of factor matrix in tensor decomposition can determine the HSI compression performance. It is worth noting that HSI is highly correlated in bands. However, this phenomenon is ignored by the previous TD method. Aiming at improving the HSI compression performance, we propose a method called correlation-based TD initialization algorithm. As HSI is well approximated by means of a reference band. In accordance with the SVD result of the reference band, the initialized factor matrices of TD are produced. We compare our methods with random and SVD-based initialization methods. The experimental results reveal that our correlation-based TD initialization method is capable of significantly reducing the computational cost of TD while keeping the initialization quality and compression performance.
\end{abstract}

\section{Index Terms}

Tensor decomposition (TD); hyperspectral image compression; initialization.

\section{INTRODUCTION}

With the wide applications of hyperspectral imaging technology, hyperspectral image (HSI) appears in many fields such as: remote sensing [Fang et al.(2017)Fang, Wang, Li, and Benediktsson], diagnostic medicine [Akbari et al.(2010)Akbari, Kosugi, Kojima, and Tanaka], image

This work is supported in part by the Open Research Fund of Key Laboratory of Spectral Imaging Technology, Chinese Academy of Sciences (Grant No. LSIT201606D) and the Open Project Program of the National Laboratory of Pattern Recognition (NLPR) (Grant No. 201800030).

Rui Li is with the School of Electronic and Information Engineering, Xi'an Jiaotong University, Xi'an, P. R. China, 710049.

Zhibin Pan is with the School of Electronic and Information Engineering, Xi'an Jiaotong University, Xi'an, P. R. China, 710049, (e-mail: zbpan@mail.xjtu.edu.cn).

Yang Wang is with the School of Electronic and Information Engineering, Xi'an Jiaotong University, Xi'an, P. R. China, 710049 . 
classification [Zhang et al.(2016)Zhang, Zhang, Jiao, Liu, Wang, and Hou], unmixing [Mei] et al.(2018)Mei, Ma, Li, Fan, Huang, and Ma], and more [Coutinho et al.(2017)Coutinho, Cintra, and Bayer], [SShi and Pun(2017)], [Md Noor et al.(2017)Md Noor, Ren, Marshall, and Michael], [Ma et al.(2018)Ma, Li, Li, Mei, and Ma]. The HSI is composed by plenty of bands which are images of the same scene on different wavebands, Therefore, the bands of HSIs share the similar characteristics. However, hyperspectral imaging suffers from many issues. One major issue is that the large amount of data makes HSI difficult to transmit and process. In addition, noise and limited illumination in individual band are two problems. Aiming at addressing the mentioned issues, Tucker Decomposition (TD) is presented [Tucker(1966)], [Karami et al.(2012)Karami, Yazdi, and Mercier], [Wang et al.(2017)Wang, Lin, Zhao, Yue, Meng, and Leung], [Sidiropoulos et al.(2017)Sidiropoulos, De Lathauwer, Fu, Huang, Papalexakis, and Faloutsos], [Zeng et al.(2017)Zeng, Zhang, and Bai]. The HSI is considered as a tensor while the decomposition results are factor matrices and a core tensor. The sizes of core tensor in TD should be smaller than original corresponding sizes. Accordingly, the number of eigenvectors is reduced. HSIs are significantly compressed.

As traditional method tackles the spatial domain, the strong correlation between bands is ignored [Dragotti et al.(2000)Dragotti, Poggi, and Ragozini], consequently, the compression performance of these methods is compromised for this drawback. One advantage of TD is that it learns a low dimensional representation. The spatial and spectral correlations are simultaneously addressed. The vector in factor matrices depicts the low dimension embedding of a corresponding slice of tensor [Jiang et al.(2018)Jiang, Ding, Tang, and Luo]. Thus, the decomposition solves the spectral correlation in HSI well. However, there are some issues needing to be settled. The TD is solved by alternating least square (ALS) method. Although the alternating minimization in TD is nonconvex, the objective function is strictly convex with respect to one set of variables [Cichocki et al.(2008)Cichocki, Zdunek, and Amari]. The ALS method is influenced by many issues [Da Silva Fernandes et al.(2017)Da Silva Fernandes, Tork, and da Gama], one critical issue is that the initial factor matrices are required. The convergence rate of TD is remarkably influenced by the qualities of initial factor matrices. A well designed initial factor matrix can make sure the convergence of iterative strategy and reduce the number of iterations. Otherwise, the algorithm may get stuck in a local minimum, for some data the convergence could be very slow when factor matrices are ill-conditioned or when collinearity occurs in the columns of these matrices [Cichocki et al.(2008)Cichocki, Zdunek, and Amari]. 


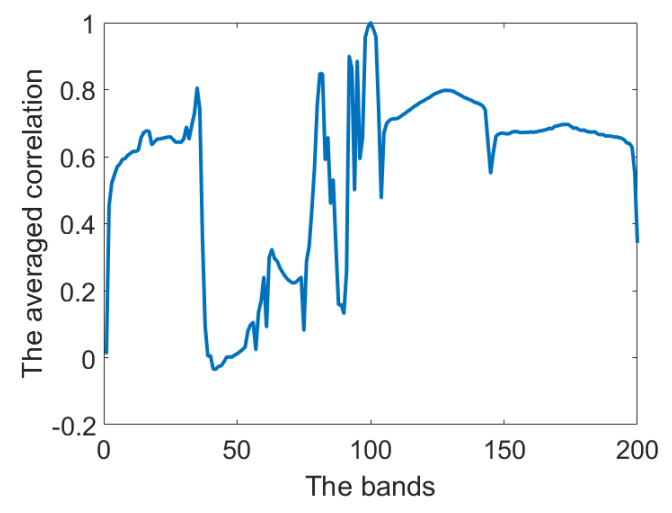

(a) The averaged correlation of all bands in Indian pines.

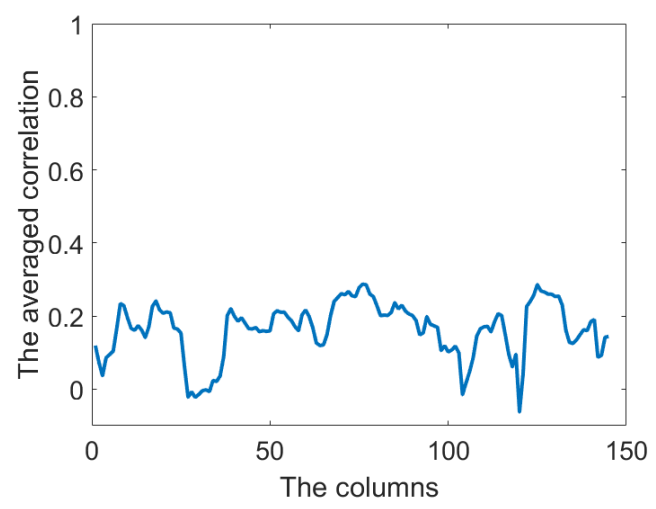

(b) The averaged correlation of all columns in the 50th band of Indian pines.

Fig. 1. The comparison of spectral correlation and spatial correlation in Indian pines.

There are two kinds of initialization method for TD. The first method is random method, which generates orthogonal vectors of factor matrix randomly. The factor matrix is columnwise orthonormal. The random method is easy to implement, but the TD may converge in many iterations, and the convergence of the TD is not guaranteed. The second method resorts singular vector decomposition (SVD) to tackle the factor matrices. The factor matrices associated to each mode are obtained by applying SVD on the corresponding matricization of the tensor.

However, these two initialization methods are neither effective nor addressed for HSIs. Fig.1 shows that HSI has stronger spectral correlation, which implies the band in HSIs can be well represented by other bands. To the best of our knowledge, there is no study in literature utilize the spectral correlation in HSI. We can utilize the spectral correlation to design a new TD initialization method for HSI to improve the compression performance and reduce the time consumption.

The highlights of this paper are listed below:

- We first explain the factor matrices in TD can be well approximated by the SVD results of some reference band;

- According to our conclusion we give our correlation-based initialization method for TD;

- We test our method to compress the HSI and achieve considerable results.

The rest of this paper are organized as follows: The basic knowledge of tensor analysis is introduced in Section 2. We prove that the factor matrices in TD can be well approximated by 
the SVD results of our reference band, and then propose our initialization method in Section 3. The experimental results are given in Section 4. We finally conclude our method in Section 5.

\section{TENSOR PRELIMINARY}

Before we introduce our method, we give some preliminaries of tensor analysis to help understand our method in this section. The notations used in this paper are summarized in the table below.

TABLE I

Notations ABOUt TENSOR

\begin{tabular}{cc}
\hline & Notations used in this paper \\
\hline $\mathcal{X} \in \mathbb{R}^{I_{1} \times I_{2} \times \cdots \times I_{N}}$ & Kronecker product \\
$x\left(i_{1}, i_{2}, \ldots, i_{N}\right)$ & A $N_{t h}$-order tensor \\
$\mathbf{X}_{(1)}$ & The entry of tensor $\mathcal{X} \in \mathbb{R}^{I_{1} \times I_{2} \times \cdots \times I_{N}}$ \\
$\mathbf{X}^{I \times J K}$ & The mode-1 matricization of $\mathcal{X}$ \\
$\mathbf{U}^{(i)}$ & A matrix of size $I \times J K$ \\
$\mathbf{X}_{(i)}^{k}$ & $i_{t h \text {-order }}$ \\
$\mathbf{X}_{:: i}$ & The $k_{t h}$ slice along mode- $i$ in tensor $\mathcal{X}$ \\
\|\|$_{F}$ & The $i_{t h}$ slice in tensor $\mathcal{X}$ \\
& The Frobenius norm of a tensor $\mathcal{X}$ \\
\hline
\end{tabular}

In multilinear algebra, Tensor is actually a multidimensional array. We denote $N_{t h}$-order tensors by bold uppercase mathcal letters $\mathcal{X} \in \mathbb{R}^{I_{1} \times I_{2} \cdots \times I_{N}}$. The order of the tensor is also referred as mode. The first-order tensor is known as vector and the second-order tensor is known as matrix. The mode-n matricization means rearranging the tensor as matrix by fixing the $n_{t h}$-order index and varying other indices. It is also a flattening process in Fig. 2. For a third-order tensor $\mathcal{X} \in \mathbb{R}^{I \times J \times K}$, there are three mode-n matricizations.

The mode-1 Kiers matricization [Kolda and Bader(2009)] of a third-order tensor $\mathcal{X}$ results in $\mathbf{X}_{(1)} . \mathbf{X}_{(2)}$ and $\mathbf{X}_{(3)}$ are the mode-2 and mode-3 Kiers matricization results, respectively. The matricizations in this paper are all Kiers matricizations.

The Frobenius norm of a tensor is defined as follow:

$$
\|\mathcal{X}\|_{F}=\sqrt{\langle\mathcal{X}, \mathcal{X}\rangle}=\left(\sum_{i_{1}=1}^{I_{1}} \sum_{i_{2}=1}^{I_{2}} \cdots \sum_{i_{n}=1}^{I_{N}}\left|x_{i_{1} i_{2} \ldots i_{n}}\right|^{2}\right)^{1 / 2}
$$




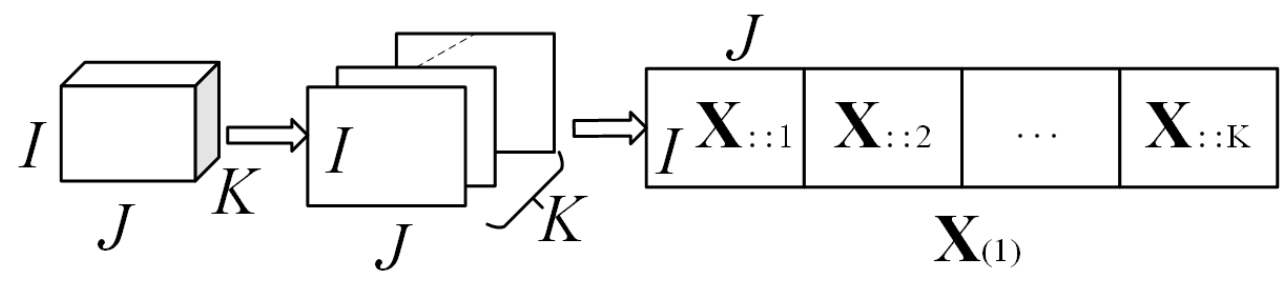

Fig. 2. The mode-1 matricization is obtained by flattening the tensor along its first mode.

The mode-n tensor-matrix product of a tensor $\mathcal{X} \in \mathbb{R}^{I_{1} \times I_{2} \times \cdots \times I_{N}}$ multiplied by matrix $\mathbf{U} \in$ $\mathbb{R}^{J_{n} \times I_{n}}$ is denoted as $\mathcal{X} \times{ }_{n} \mathbf{U}$ :

$$
\mathcal{X} \times{ }_{n} \mathbf{U}_{i_{1}, i_{2}, \ldots, i_{n-1}, i_{n}, i_{n+1}, \ldots, i_{N}}=\sum_{i_{n}=1}^{I_{n}} a_{i_{1} i_{2} \ldots i_{N}} \times u_{i_{n} j_{n}}
$$

where the height of matrix $\mathbf{U}$ should be the same with the mode-n size of tensor $\mathcal{X}$, this multiplication changes the mode-n size of tensor $\mathcal{X}$ from $I_{n}$ to $J_{n}$.

We can use this kind of multiplication to change the sizes of a tensor.

$$
\boldsymbol{X}=\mathcal{A} \times{ }_{1} \mathbf{U}^{(1)} \times{ }_{2} \mathbf{U}^{(2)} \cdots \times{ }_{N} \mathbf{U}^{(N)}
$$

where the width of matrix $\mathbf{U}^{(n)}$ should be equal to the size of mode-n of tensor $\mathcal{A}$.

Through the above equations, We can introduce the decomposition of third-order tensor in this paper

$$
\mathcal{X}=\mathcal{G} \times{ }_{1} \mathbf{U}^{(1)} \times{ }_{2} \mathbf{U}^{(2)} \times{ }_{3} \mathbf{U}^{(3)}
$$

where $\mathcal{X}$ is the original tensor, and $\mathcal{G}$ is the core tensor of $\mathcal{X}, \mathbf{U}^{(1)}, \mathbf{U}^{(2)}, \mathbf{U}^{(3)}$ are factor matrices where $\mathbf{U}^{(n)} \mathbf{U}^{(n) T}=I_{J_{n}}, n=1,2,3$. the core tensor can also be obtained by the reverse progress, which is given below:

$$
\mathcal{G}=\mathcal{X} \times{ }_{1} \mathbf{U}^{(1) T} \times{ }_{2} \mathbf{U}^{(2) T} \times{ }_{3} \mathbf{U}^{(3) T}
$$

\section{CORRELATION-BASEd Initialization Method FOR TD}

Before we start our discussion about TD, we should know that TD is resolved by alternating least square (ALS) method, a third-order tensor is flattened along each modes as three matricizations, the initialized factor matrices are obtained by SVD results of matricizations. We will discuss the correlation in HSI and give a more efficient initialization method. 
In this section, we first build a model of matricization and show that mode- 1 and mode2 matricizations can be well approximated a reference band. As the factor matrices $\mathbf{U}^{(1)}, \mathbf{U}^{(2)}$ are the SVD results of mode-1 and mode-2 matricizations respectively, we resort the SVD result of reference band to approximate $\mathbf{U}^{(1)}, \mathbf{U}^{(2)}$. Then, according our discussion, we give our correlation-based initialization method.

\section{A. Building a model of matricization}

Considering the HSI $\mathcal{X} \in \mathbb{R}^{I_{1} \times I_{2} \times I_{3}}$ is a special tensor in which the bands are highly similar, the mode-1 matricization is actually a matrix stitched by $I_{1}$ slices of tensor. The Fig. 2 depicts this mode-1 matricization. The SVD-based initialization method is essentially implementing SVD on each mode-n matricization $\mathbf{X}_{(n)}$ and seeking an orthonormal factor matrix $\mathbf{U}^{(n)}$. The factor matrix is composed by the leading singular vectors of $\mathbf{X}_{(n)}$. Since the slices in $\mathbf{X}_{(1)}$ are the bands of HSI, these bands are highly correlated. The Fig. 1 shows this phenomenon. Intuitively, we can conclude that the SVD result of $\mathbf{X}_{(1)}$ should be highly relevant to the SVD result of the slices in $\mathbf{X}_{(1)}$. To verify our guess, we can build a model to modify the process.

We use a band which can represent the bands in HSI $\mathcal{X} \in \mathbb{R}^{I_{1} \times I_{2} \times I_{3}}$ best as the reference band $\overline{\mathbf{X}}$. The method to achieve reference band is given in the following subsection.

The mode-1 and mode-2 matricizations $\mathbf{X}_{(1)}, \mathbf{X}_{(2)}$ can be expressed as:

$$
\begin{gathered}
\mathbf{X}_{(1)}=\boldsymbol{i}_{1 \times P} \otimes \overline{\mathbf{X}}+\mathbf{e}_{1}, \\
\mathbf{X}_{(2)}=\boldsymbol{j}_{1 \times P} \otimes \overline{\mathbf{X}}^{T}+\mathbf{e}_{2} .
\end{gathered}
$$

In the equations above, $\mathbf{e}$ is the approximation error, $\mathbf{X}_{(1)}, \mathbf{X}_{(2)}$ are approximated as the Kronecker products of a vector and the reference band $\overline{\mathbf{X}} . \boldsymbol{i}_{1 \times P}$ and $\boldsymbol{j}_{1 \times P}$ are the approximation coefficient vectors. $\mathbf{X}_{(3)}$ cannot be approximated by $\overline{\mathbf{X}}$ for each band is viewed as a column vector in $\mathbf{X}_{(3)}$. The reference band $\overline{\mathbf{X}}$ can be decomposed by SVD:

$$
\overline{\mathbf{X}}=\mathbf{U} \boldsymbol{\Sigma} \mathbf{V}^{T}
$$

Substituting $\overline{\mathbf{X}}$ into Eq. 6, it is equivalent to

$$
\mathbf{X}_{(1)}=\boldsymbol{i}_{1 \times P} \otimes\left(\mathbf{U} \boldsymbol{\Sigma} \mathbf{V}^{T}\right)+\mathbf{e}_{1}
$$

Since the Kronecker product has the property below:

$$
(\mathbf{A} \otimes \mathbf{B})(\mathbf{C} \otimes \mathbf{D})(\mathbf{E} \otimes \mathbf{F})=(\mathbf{A C E}) \otimes(\mathbf{B D F})
$$


The vector $\boldsymbol{i}_{1 \times P}$ can be represented as:

$$
\boldsymbol{i}_{1 \times P}=1 \times \boldsymbol{\alpha}^{T} \times \mathbf{B}
$$

where $\boldsymbol{\alpha}^{T}$ is a vector, its first entry is 1 , and other entries are 0 . The first row of matrix $\mathbf{B}$ is $\boldsymbol{i}_{1 \times P}$. Then, we can derive:

$$
\mathbf{X}_{(1)}=\mathbf{U}\left(\boldsymbol{\alpha}^{T} \otimes \boldsymbol{\Sigma}\right)\left(\mathbf{B} \otimes \mathbf{V}^{T}\right)+\mathbf{e}_{1}
$$

For $\mathbf{X}_{(2)}$, we can derive the similar result, where $\overline{\mathbf{X}}$ is transposed.

$$
\mathbf{X}_{(2)}=\mathbf{V}(\boldsymbol{c} \otimes \boldsymbol{\Sigma})\left(\mathbf{D}^{T} \otimes \mathbf{U}^{T}\right)+\mathbf{e}_{2}
$$

Therefore, the SVD on $\mathbf{X}_{(1)}$ results in the equation above. In other words, the SVD-based initialization method obtains the same left singular matrix as $\overline{\mathbf{X}}$. We can obtain the same result on $\mathbf{X}_{(2)}$, because $\mathbf{X}_{(2)}$ and $\mathbf{X}_{(1)}$ are both approximated by $\overline{\mathbf{X}}$. These two matricizations are similar because they are the bands spliced in different directions. Thus, the SVD-based initialization method is doing SVD on the duplications of $\overline{\mathbf{X}}$. The $\mathbf{X}_{(3)}$ cannot be handled in this way, because the band is viewed as vector and spatial correlation is not considered in this matricization, thus the SVD on the reference band is not correlated with the SVD on $\mathbf{X}_{(3)}$. Our method to obtain the factor matrix $\mathbf{U}^{(3)}$ is the same with the SVD-based initialized method.

\section{B. Correlation-based initialization method}

Our method is inspired by the high correlation of bands in HSIs. According to the model we build, when $\mathbf{X}_{(1)}$ can be well represented by $\overline{\mathbf{X}}$, the SVD results on $\mathbf{X}_{(1)}$ and $\overline{\mathbf{X}}$ are also relevant. Since the spectral correlation of HSIs is strong, we can conclude that $\mathbf{X}_{(1)}$ can be well represented by $\overline{\mathbf{X}}$. We present our method in this section.

The reference band $\overline{\mathbf{X}}$ is critical important in this method, the $\overline{\mathbf{X}}$ should well represent the bands in $\mathcal{X} \in \mathbb{R}^{I_{1} \times I_{2} \times I_{3}}$. To obtain such a reference band, the representative ability and the computational cost are both considered as the rules of our method. Thus, we recommend adopt the mean band as the reference band.

$$
\boldsymbol{X}_{3}=\mathcal{X} \times_{1} \mathbf{U}^{(1)} \times_{2} \mathbf{U}^{(2)}
$$




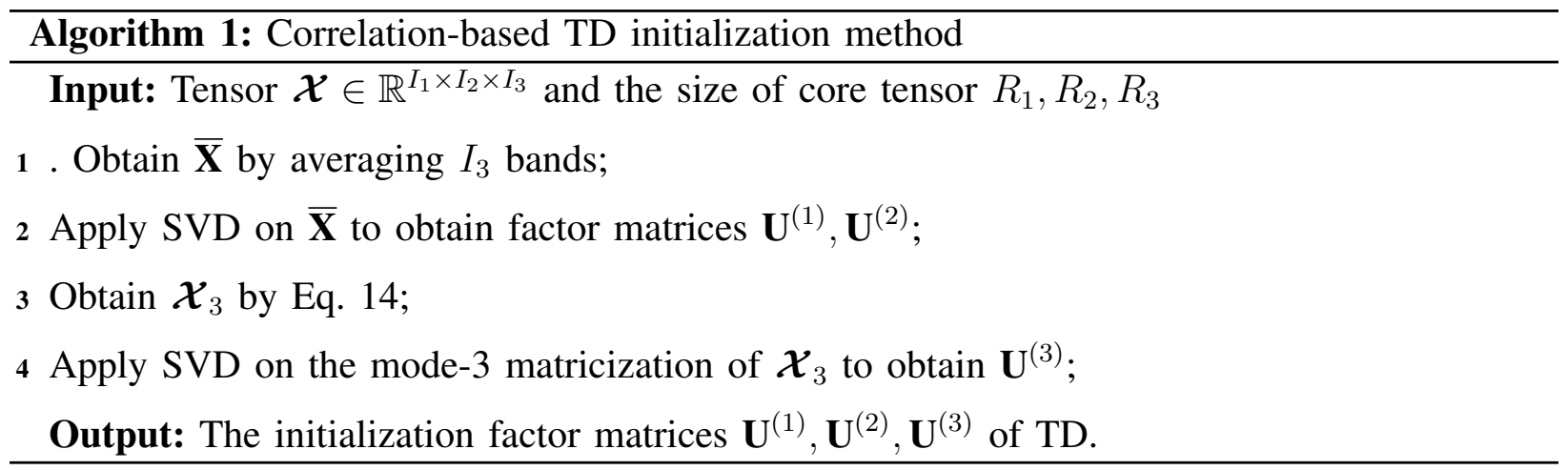

\section{Computational cost}

The computational cost of SVD is related to the matrix size. There are $M N^{2}$ times multiplication operations for applying SVD on a $M \times N$ matrix, thus, we need $I_{1}\left(I_{2} I_{3}\right)^{2}$ multiplication operations for applying SVD on the mode-1 matricization of tensor $\mathcal{X} \in \mathbb{R}^{I_{1} \times I_{2} \times I_{3}}$. But the multiplication operations are much less in our initialization method for the size of reference band is $I_{1} \times I_{2}$, which is only the size of one slice in mode-1 matricization. Moreover, we can obtain initial factor matrices $\mathbf{U}^{(1)}, \mathbf{U}^{(2)}$ by applying SVD once, especially the computational cost of applying SVD on a mode-n matricization is large. It is obvious that we can save computational cost by averaging the bands to obtain an averaged band, the final computational cost of our method is $I_{1} I_{2}^{2}+I_{3}\left(I_{1} I_{2}\right)^{2}$, where $I_{3}\left(I_{1} I_{2}\right)^{2}$ is the computational cost of $\mathbf{U}^{(3)}$, while the SVDbased method takes $I_{3}\left(I_{2} I_{1}\right)^{2}+I_{1}\left(I_{2} I_{3}\right)^{2}+I_{2}\left(I_{3} I_{1}\right)^{2}$ times multiplication. Considering there are usually hundreds of bands in HSIs, which means $I_{3}$ is large, thus, the term of $I_{1} I_{2}^{2}$ is negligible compared to other terms. In other words, the computational cost of our method is concentrated on the solving of factor matrix $\mathbf{U}^{(3)}$. The results in Table 3 and Table 4 verify our conclusion.

\section{EXPERIMENTAL RESULTS}

To verify the compression performance and time consumption of our proposed correlationbased TD initialization method, the experiments were carried out using MATLAB 2015b along with Tensor Toolbox [Bader et al.(2015)Bader, Kolda et al.] on a machine of Intel Core i7 6700 processor and 16GB RAM. We show our compression performance, initialization results and time consumption in this section. 


\section{A. Datasets and evaluation metrics}

We consider two HSIs in our experiments: Indian Pines and Salinas, which are obtained by Airborne Visible/Infrared Imaging Spectrometer (AVIRIS) sensor. The sizes are $145 \times 145 \times$ $200,512 \times 217 \times 224$, respectively the first two dimensionalities are spatial sizes and the third dimensionality is the spectral size. These HSIs are obtained on a spectral range of 400-2500 nm.

The fitness of Tucker decomposition is defined as the Frobenius norm of the original tensor and reconstructed tensor. It is given as follow:

$$
\text { Fitness }=1-\left\|\mathcal{X}-\mathcal{X}_{\text {rec }}\right\|_{F} /\|\mathcal{X}\|_{F} \text {. }
$$

We compare the initialization time, the number of iterations in TD and the total time consumption of the TD which includes the initialization time and iteration time.

\section{B. Results}

We compare our method with three compression method: principal component analysis (PCA)+JPEG2000, three Dimensional-Set Partitioned Embedded bloCK (3D-SPECK) and multilinear principal component analysis (MPCA). PCA+JPEG2000 is a traditional compression method applied on HSI, 3D-SPECK is a commonly used method based on wavelet, MPCA is multilinear algebra method that can compress spectral and spatial redundancy simultaneously. These three methods are representative in three areas. Our method benefits from the TD, can well compress the HSI. The results are list in Table 2. The results show that our proposed method achieves the best compression performance.

To verify the initialization performance of our method, we test our method against random initialization method and SVD-based initialization method. The sizes of core tensor are all setting as the half of each corresponding dimensionalities. The results in Table 3 and Table 4 show that our initialization method consumes less time than random and SVD-based initialization methods, while the final compression performances are same (which is listed in Table 2).

To further evaluate the initialization performance of our method, we compare our method with SVD-based method on Indian Pines. The time to obtain each factor matrix is listed in Table 5. Our method obtains the first two factor matrices $\mathbf{U}^{(1)}, \mathbf{U}^{(2)}$ in 0.625 seconds while the SVDbased method needs 199.376 seconds in total. Thus, this time consumption of 0.625 second can be ignored and most of time is consumed for computing the $\mathbf{U}^{(3)}$ in our method, which is still much less than the SVD-based method. Although, we carry out SVD on a mode-3 matricization, 
TABLE II

THE SNR (DB) OF OUR PROPOSED METHOD IS COMPARED WITH THREE METHODS WITH BR VARYING FROM 0.1 BPPPB TO 0.4 ВРPPB

\begin{tabular}{lccccc}
\hline & & PCA+JPEG2000 & 3D-SPECK & MPCA & Our method \\
\hline \multirow{4}{*}{ Indian Pines } & 0.1 & $\mathbf{3 4 . 2 1}$ & 27.02 & 33.58 & 34.16 \\
& 0.2 & 36.54 & 30.53 & 36.72 & $\mathbf{3 6 . 8 9}$ \\
& 0.3 & 37.51 & 32.71 & 37.98 & $\mathbf{3 8 . 2 4}$ \\
& 0.4 & 39.12 & 35.64 & 38.51 & $\mathbf{4 0 . 3 3}$ \\
\hline \multirow{4}{*}{ Salinas } & 0.1 & $\mathbf{3 4 . 8 7}$ & 29.12 & 34.21 & 34.36 \\
& 0.2 & 36.65 & 31.57 & 36.67 & $\mathbf{3 7 . 7 1}$ \\
& 0.3 & 38.12 & 33.54 & 37.55 & $\mathbf{3 9 . 6 4}$ \\
& 0.4 & 39.41 & 34.84 & 38.21 & $\mathbf{4 1 . 1 5}$ \\
\hline
\end{tabular}

TABLE III

THE COMPARATIVE RESULTS OF THREE INITIALIZATION METHODS ON INDIAN PINES.

\begin{tabular}{lccc}
\hline & Random method & SVD-based method & Our method \\
\hline Initialization time (s) & - & 1.502 & $\mathbf{0 . 2 7 0}$ \\
Number of iterations & 4 & 2 & 2 \\
Convergence time (s) & 2.940 & $\mathbf{1 . 5 0 1}$ & 1.750 \\
Total time (s) & 2.940 & 3.003 & $\mathbf{2 . 0 2 0}$ \\
\hline
\end{tabular}

TABLE IV

THE COMPARATIVE RESULTS OF THREE INITIALIZATION METHODS ON SALINAS.

\begin{tabular}{lccc}
\hline & Random method & SVD-based method & Our method \\
\hline Initialization time (s) & - & 32.175 & $\mathbf{3 . 0 6 6}$ \\
Number of iterations & 4 & 2 & 2 \\
Convergence time (s) & 40.427 & $\mathbf{3 0 . 3 0 6}$ & 33.373 \\
Total time (s) & 40.427 & 62.481 & $\mathbf{3 6 . 4 4 0}$ \\
\hline
\end{tabular}


TABLE V

THE TIME (S) CONSUMPTION OF COMPUTING THREE FACTOR MATRICES IN 100 TIMES.

\begin{tabular}{lcc}
\hline Factor matrix & SVD-based method & Our method \\
\hline $\mathbf{U}^{(1)}$ & 93.438 & $\mathbf{0 . 6 2 5}$ \\
$\mathbf{U}^{(2)}$ & 105.938 & \\
$\mathbf{U}^{(3)}$ & 110.780 & $\mathbf{4 1 . 1 2 5}$ \\
Total cost & 310.156 & $\mathbf{4 1 . 7 5 0}$ \\
\hline
\end{tabular}

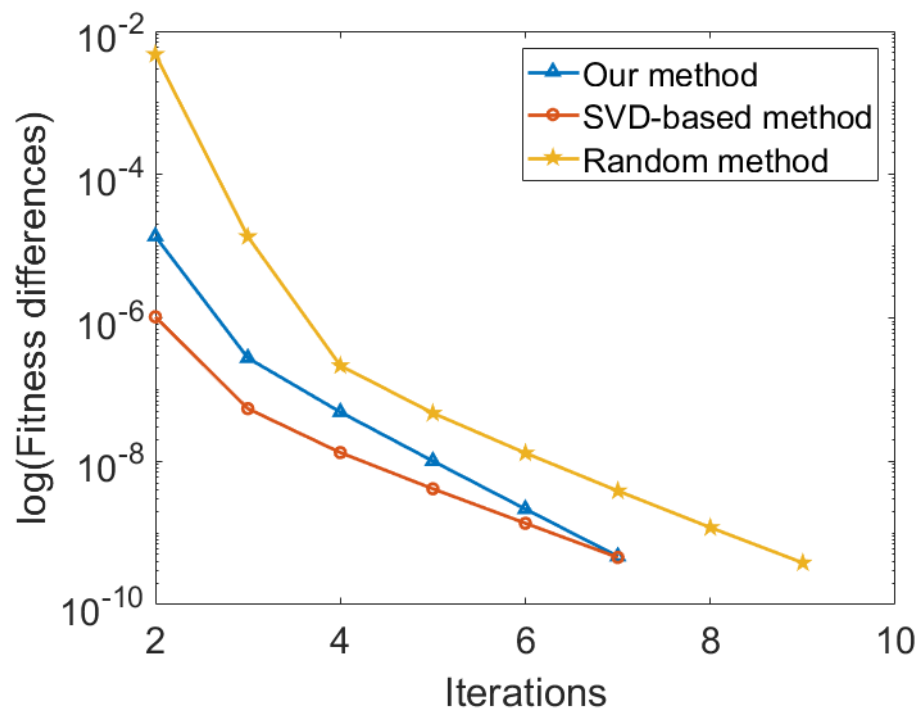

Fig. 3. The fitness difference varies with the iterations.

the matricization is obtained by the original tensor rotated by the first two factor matrices and the sizes of tensor become smaller, so that the SVD consumes less time in our method.

\section{Iterations and fitness}

We also compare the fitness of three method varying with iterations. As the fitness of random method is much lower than our method and SVD-based method, we show the curve from the second iteration. Fig. 3 shows that our method has a fitness close to SVD-based method and much better than random method. Our method saves nearly three iterations compared to the random method. We compare the fitness difference of each iteration and the curve in logarithmic coordinate system shows that our method has a performance close to SVD-based method.

We also list the fitness differences of first three iterations in Table 6 for comparison. The 
TABLE VI

THE FITNESS DIFFERENCES OF THREE METHODS IN FIRST THREE ITERATIONS.

\begin{tabular}{lccc}
\hline Iteration & SVD-based method & Random method & Our method \\
\hline 1 & $1.034 \mathrm{e}-6$ & $4.712 \mathrm{e}-3$ & $1.374 \mathrm{e}-5$ \\
2 & $5.423 \mathrm{e}-8$ & $1.363 \mathrm{e}-5$ & $2.761 \mathrm{e}-7$ \\
3 & $1.321 \mathrm{e}-8$ & $2.158 \mathrm{e}-7$ & $4.855 \mathrm{e}-8$ \\
\hline
\end{tabular}

results show the fitness changing of our method is close to the SVD-based method and much smaller than Random method. This verifies that our method can obtain the initialized factor matrices close to the best initialized factor matrices with a much lower computational cost.

\section{CONCLUSION}

We utilize the strong correlation between the bands of HSIs and propose a new initialization method for TD, we prove that mode-1 and mode- 2 matricizations of HSI can be well approximated by the Kronecker product of a reference band and an approximation vector. According to this conclusion, we propose our correlation-based initialization algorithm for tensorbased HSI compression. Our method ensures the TD method can converge in a few iterations and the computational cost is obviously saved. The experiments verify the good initialization performance, compression performance and less time consumption of our method.

\section{ACKNOWLEDGEMENT( $S$ )}

This work is supported in part by the Open Research Fund of Key Laboratory of Spectral Imaging Technology, Chinese Academy of Sciences (Grant No. LSIT201606D) and the Open Project Program of the National Laboratory of Pattern Recognition (NLPR) (Grant No. 201800030).

\section{REFERENCES}

[Akbari et al.(2010)Akbari, Kosugi, Kojima, and Tanaka] Akbari H, Kosugi Y, Kojima K, Tanaka N (2010) Detection and analysis of the intestinal ischemia using visible and invisible hyperspectral imaging. IEEE Transactions on Biomedical Engineering 57(8):2011-2017

[Bader et al.(2015)Bader, Kolda et al.] Bader BW, Kolda TG, et al. (2015) Matlab tensor toolbox version 2.6. available online. URL: http://www sandia gov/tgkolda/TensorToolbox

[Cichocki et al.(2008)Cichocki, Zdunek, and Amari] Cichocki A, Zdunek R, Amari Si (2008) Nonnegative matrix and tensor factorization [lecture notes]. IEEE Signal Processing Magazine 25(1):142-145 
[Coutinho et al.(2017)Coutinho, Cintra, and Bayer] Coutinho VdA, Cintra RJ, Bayer FM (2017) Low-complexity multidimensional dct approximations for high-order tensor data decorrelation. IEEE Transactions on Image Processing 26(5):22962310

[Da Silva Fernandes et al.(2017)Da Silva Fernandes, Tork, and da Gama] Da Silva Fernandes S, Tork HF, da Gama JMP (2017) The initialization and parameter setting problem in tensor decomposition-based link prediction. In: 2017 IEEE International Conference on Data Science and Advanced Analytics (DSAA), IEEE, pp 99-108

[Dragotti et al.(2000)Dragotti, Poggi, and Ragozini] Dragotti PL, Poggi G, Ragozini AR (2000) Compression of multispectral images by three-dimensional spiht algorithm. IEEE Transactions on Geoscience and Remote Sensing 38(1):416-428

[Fang et al.(2017)Fang, Wang, Li, and Benediktsson] Fang L, Wang C, Li S, Benediktsson JA (2017) Hyperspectral image classification via multiple-feature-based adaptive sparse representation. IEEE Transactions on Instrumentation and Measurement 66(7):1646-1657

[Jiang et al.(2018)Jiang, Ding, Tang, and Luo] Jiang B, Ding C, Tang J, Luo B (2018) Image representation and learning with graph-laplacian tucker tensor decomposition. IEEE Transactions on Cybernetics 49(4):1417-1426

[Karami et al.(2012)Karami, Yazdi, and Mercier] Karami A, Yazdi M, Mercier G (2012) Compression of hyperspectral images using discerete wavelet transform and tucker decomposition. IEEE Journal of Selected Topics in Applied Earth Observations and Remote Sensing 5(2):444-450

[Kolda and Bader(2009)] Kolda TG, Bader BW (2009) Tensor decompositions and applications. SIAM Review 51(3):455-500

[Ma et al.(2018)Ma, Li, Li, Mei, and Ma] Ma Y, Li C, Li H, Mei X, Ma J (2018) Hyperspectral image classification with discriminative kernel collaborative representation and tikhonov regularization. IEEE Geoscience and Remote Sensing Letters 15(4):587-591

[Md Noor et al.(2017)Md Noor, Ren, Marshall, and Michael] Md Noor S, Ren J, Marshall S, Michael K (2017) Hyperspectral image enhancement and mixture deep-learning classification of corneal epithelium injuries. Sensors 17(11):2644

[Mei et al.(2018)Mei, Ma, Li, Fan, Huang, and Ma] Mei X, Ma Y, Li C, Fan F, Huang J, Ma J (2018) Robust gbm hyperspectral image unmixing with superpixel segmentation based low rank and sparse representation. Neurocomputing 275:2783-2797

[Shi and Pun(2017)] Shi C, Pun CM (2017) 3d multi-resolution wavelet convolutional neural networks for hyperspectral image classification. Information Sciences 420:49-65

[Sidiropoulos et al.(2017)Sidiropoulos, De Lathauwer, Fu, Huang, Papalexakis, and Faloutsos] Sidiropoulos ND, De Lathauwer L, Fu X, Huang K, Papalexakis EE, Faloutsos C (2017) Tensor decomposition for signal processing and machine learning. IEEE Transactions on Signal Processing 65(13):3551-3582

[Tucker(1966)] Tucker LR (1966) Some mathematical notes on three-mode factor analysis. Psychometrika 31(3):279-311

[Wang et al.(2017)Wang, Lin, Zhao, Yue, Meng, and Leung] Wang Y, Lin L, Zhao Q, Yue T, Meng D, Leung Y (2017) Compressive sensing of hyperspectral images via joint tensor tucker decomposition and weighted total variation regularization. IEEE Geoscience and Remote Sensing Letters 14(12):2457-2461

[Zeng et al.(2017)Zeng, Zhang, and Bai] Zeng W, Zhang X, Bai Y (2017) Method for multispectral images denoising based on tensor-singular value decomposition. Journal of Applied Remote Sensing 11(3):035019

[Zhang et al.(2016)Zhang, Zhang, Jiao, Liu, Wang, and Hou] Zhang E, Zhang X, Jiao L, Liu H, Wang S, Hou B (2016) Weighted multifeature hyperspectral image classification via kernel joint sparse representation. Neurocomputing 178:71-86

Rui Li received the B. S. and M. S. degree from Xidian University, Xi'an, P. R. China, in 2012 and 2015 respectively. He is 
currently working towards the $\mathrm{Ph}$. D. degree in School of Electronic and Information Engineering at Xi' an Jiaotong University, Xi'an, P. R. China. His research interests include vector quantization and hyper-spectral image processing.

Zhibin Pan received the B. S. degree in Information and Telecommunication Engineering in 1985 and the M. S. degree in Automation Science and Technology in 1988 from Xi' an Jiaotong University, P. R. China, respectively. He received the Ph. D. degree in Electrical Engineering in 2000 from Tohoku University, Japan. He is a professor in the Department of Information and Telecommunication Engineering, Xi' an Jiaotong University, P. R. China. His current research interests include image compression, multimedia security and object recognition.

Yang Wang received his B. S. degree from Xi'an Jiaotong University, Xi'an, P. R. China, in 2010. He is currently working towards the Ph. D. degree in School of Electronic and Information Engineering at Xi' an Jiaotong University, Xi'an, P. R. China. His research interests include image coding and image processing. 